\title{
Revisiting an agar-based plate method: What the static biofilm method can offer for biofilm research
}

\author{
Authors: Terhi Oja, Brianna Blomqvist, Kelli Buckingham- \\ Meyer, Darla Goeres, Pia Vuorela, \& Adyary Fallarero
}

NOTICE: this is the author's version of a work that was accepted for publication in Journal of Microbiological Methods. Changes resulting from the publishing process, such as peer review, editing, corrections, structural formatting, and other quality control mechanisms may not be reflected in this document. Changes may have been made to this work since it was submitted for publication. A definitive version was subsequently published in Journal of Microbiological Methods, Volume 107, December 2014, DOI\# 10.1016/j.mimet.2014.10.008

Oja, Terhi , Brianna Blomqvist, Kelli Buckingham-Meyer, Darla Goeres, Pia Vuorela, and Adyary Fallarero. Revisiting an agar-based plate method: What the static biofilm method can offer for biofilm research. Journal of Microbiological Methods. December 2014. Pages 157-160. https://dx.doi.org/10.1016/j.mimet.2014.10.008 


\title{
Revisiting an agar-based plate method: What the static biofilm method can offer for biofilm research
}

\author{
Terhi Oja \& Brianna Blomqvist: Pharmaceutical Sciences, Department of Biosciences, Abo Akademi \\ University, Turku, Finland \\ Kelli Buckingham-Meyer \& Darla Goeres: Center for Biofilm Engineering, Montana State University, \\ Bozeman, United States \\ Pia Vuorela \& Adyary Fallarero: Division of Pharmaceutical Biosciences, CDR, Faculty of Pharmacy, \\ University of Helsinki, Finland
}

\begin{abstract}
The development of biofilms in static plates was monitored. Glass coupons were placed on agar covered with filter paper, which was inoculated with suspended bacteria. The viable cell density, biofilms matrix and biomass were quantified. The method is excellent for adhesion and material studies, due to its simplicity and flexibility.
\end{abstract}

The unique properties of bacterial biofilms call for the development of reliable and specific research methods, different to the ones optimized for planktonic bacteria (Cos et al., 2010). Accordingly, various biofilm culturing methods have been developed, in which model biofilms grow under various fluid shear conditions in specific reactors. The choice of the biofilm reactor is based upon the research question under investigation (Buckingham-Meyer et al., 2007; Coenye and Nelis, 2010).

Biofilm formation in liquid cultures has been studied in detail, and it is thought to involve several distinctive phases. At the initial phase, planktonic bacteria are irreversibly attached to the surface. The attachment is followed by aggregation and proliferation of the cells. The production and accumulation of extracellular polymeric substances then result in the formation of a mature biofilm, where the cells are organized into a three-dimensional community surrounded by the biofilm matrix. At the final phase, cells return into a planktonic stage and detach from the biofilm (Dunne, 2002; Kiedrowski and Horswill, 2011; Otto, 2008).

Although bacterial biofilms may also form on surfaces that are not immersed in liquid, the solid-state growth methods are not as common as the well-studied liquid cultures. Among the reported solid-state methods are the colony biofilm model (Anderl et al., 2000) and the stat-ic biofilm method (Charaf et al., 1999). In the colony biofilm model, polycarbonate membrane filters are inoculated and regularly transferred onto fresh agar medium, while in the static biofilm method the biofilm is left to develop on a single filter-covered agar plate for the whole incubation period (Fig. 1). The static biofilm method has been shown to be useful in antimicrobial efficacy testing against Pseudomonas aeruginosa PAO1, ATCC 15442 and Staphylococcus aureus ATCC 6538 biofilms (Buckingham-Meyer et al., 2007; Charaf et al., 1999). However, the formation of the biofilms on static method plates has not been thoroughly characterized, nor its potential as a research choice fully explored.

Consequently, in this contribution we set off to gain a deeper under-standing of the development of static method biofilms. For this purpose, the kinetics of biofilm development was studied for the Gram-positive S. aureus ATCC 25923, a model biofilm-forming organism. Furthermore, the results were compared with S. aureus ATCC 6538, Staphylococcus epidermidis ATCC 35984, and the Gram-negative Escherichia coli XL-1 Blue and P. aeruginosa ATCC 15442. In all cases, biofilms were grown ex-actly according to the previously described method (Buckingham-Meyer et al., 2007). Briefly, sterile borosilicate glass coupons (diameter $1.27 \mathrm{~cm}$, height 0.4 $\mathrm{cm}$, BioSurface Technologies Corporation) were placed on an agar plate covered with an inoculated filter paper (Whatman Qualitative Grade 2, 70-mm diameter, GE Healthcare). The purpose of the filter paper is to provide a barrier between the cou-pons and the agar surface that allows for diffusion of nutrients while 


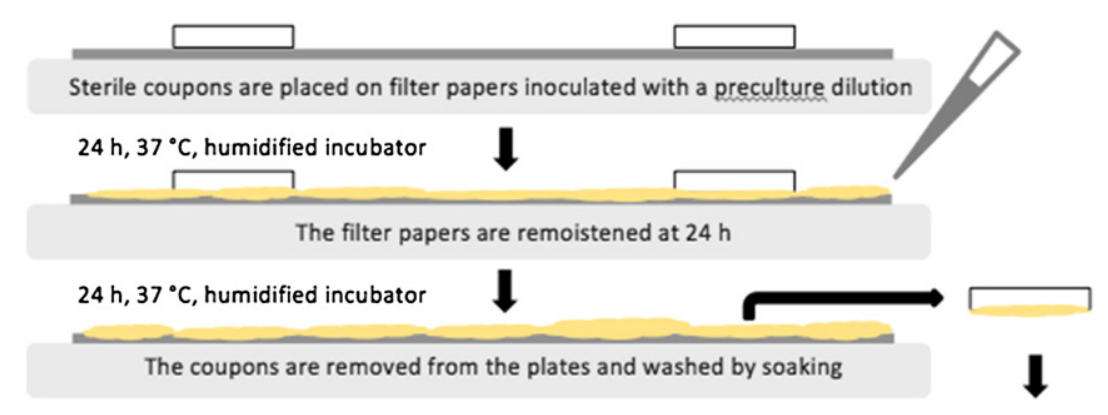

Analysis of established biofilms.

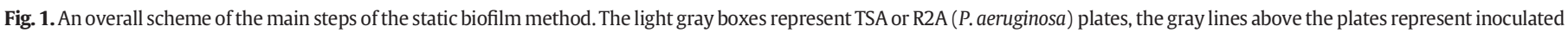
filter papers, and the coupons on the filter papers are drawn as empty boxes. The biofilms are drawn in yellow. Only the original 48 -h incubation period is shown here.

preventing the agar from sticking to the coupon surface. The inoculum consisted of $1.5 \mathrm{~mL}$ of a 1:10 dilution of an 18-24 h old preculture in either tryptone soy broth (TSB, Sigma-Aldrich) or in a 100 -fold diluted TSB (only for P. aeruginosa ATCC 15442). After $24 \mathrm{~h}$ of incubation in a humidified incubator at $37^{\circ} \mathrm{C}$, the filters were remoistened with $1.5 \mathrm{~mL}$ of 10 -fold or 1000 -fold (P. aeruginosa ATCC 15442) diluted TSB (Fig. 1, Buckingham-Meyer et al., 2007).

In this contribution, longer incubation periods for growing the biofilm were studied $(1,2,4,24,32$ or $48 \mathrm{~h})$, and the coupons were analyzed by either viable cell counts or crystal violet (CV) staining of the total biomass. In the case of S. aureus ATCC 25923 the coupons were additionally analyzed by staining with a wheat germ agglutinin (WGA) Alexa Fluor 488 conjugate (Molecular Probes, Thermo Fisher Scientific). At the end of the selected incubation period, each coupon was rinsed by dipping it in TSB, and subsequently analyzed according to a specific protocol. Differences in the protocols were only owed to practical reasons, as the work with three of the strains (S. aureus ATCC 25923, S. epidermidis ATCC 35984 and E. coli XL-1 Blue) was performed in Finland while the other strains (S. aureus ATCC 6538 and $P$. aeruginosa ATCC 15442) were studied in the United States.

For viable cell counting, the biofilms (except those formed by S. aureus ATCC 6538 and $P$. aeruginosa ATCC 15442) were removed and dispersed by a sonication-based method, simpler than the one originally reported (Buckingham-Meyer et al., 2007; Charaf et al., 1999), which was based on scraping biofilm off the surface. Coupons were fully immersed in $1 \mathrm{~mL}$ of $0.5 \%$ (wt./vol.) Tween 20 (Sigma-Aldrich) in TSB, followed by quick vigorous mixing and 5 min of sonication in a water bath sonicator (Sonorex Digitec, Bandelin) at $25{ }^{\circ} \mathrm{C}, 35 \mathrm{kHz}$. In the case of S. aureus ATCC 6538 and P. aeruginosa ATCC 15442 biofilms, a previously published protocol was followed (ASTM International, 2013). Briefly: each coupon was fully immersed in a conical vial containing $10 \mathrm{~mL}$ of buffered water $\left(\mathrm{KH}_{2} \mathrm{PO}_{4} 0.3 \mathrm{mM}, \mathrm{MgCl} 6 \mathrm{H}_{2} \mathrm{O} 2.0 \mathrm{mM}\right.$; pH 7.2). The vial was vortexed ( $30 \mathrm{~s}$ ) and sonicated $\left(30 \mathrm{~s}, 45 \mathrm{kHz}, 25^{\circ} \mathrm{C}\right)$ in 2 cycles followed by one more vortexing step for $30 \mathrm{~s}$. In all cases, dilution series of the resulting suspensions were prepared and plated for the determination of the colony forming units (CFUs). All plates were incubated at $37^{\circ} \mathrm{C}$ for $24 \mathrm{~h}$.

Alternatively, the washed coupons with intact biofilms were analyzed by staining with CV or the WGA-Alexa fluor conjugate. For all biofilms except those formed by S. aureus ATCC 6538 and P. aeruginosa ATCC 15442, the following modifications were made to a previously described CV protocol (Sandberg et al., 2008). The coupons with the intact biofilm side up were transferred to a 12-well plate, and gently covered with $70 \mu \mathrm{L}$ of $2.3 \%$ (wt./vol.) CV solution (Sigma-Aldrich) for $5 \mathrm{~min}$. This volume was enough to cover the surface of the glass coupon where the biofilm had formed. Washing was done three times with $3 \mathrm{~mL}$ of Milli-Q water, and the bound dye was dissolved in $1.5 \mathrm{~mL}$ of $96 \%(\mathrm{vol} / \mathrm{vol}$ ) ethanol $(1 \mathrm{~h})$. In the case of the intact, washed $S$. aureus ATCC 6538 and P. aeruginosa ATCC 15442 biofilms, the staining was performed for $15 \mathrm{~min}$ in $5 \mathrm{~mL}$ of $0.5 \%$ (wt./vol.) CV (Acros) in Milli-Q water, followed by washing three times in $30 \mathrm{~mL}$ of sterile buffered water. The dye was dissolved in $5 \mathrm{~mL}$ of $95 \%$ ( $\mathrm{vol} / \mathrm{vol}$ ) ethanol (10 $\mathrm{min}$ ).

For WGA staining of the S. aureus ATCC 25923 biofilms, a previously described protocol (Skogman et al., 2012) was followed with minor modifications: the coupons were washed three times in a 12 -well plate with $3 \mathrm{~mL}$ of PBS, and the dye was dissolved in $1 \mathrm{~mL}$ of $33 \%$ acetic acid.

The results revealed that for S. aureus ATCC 25923 there were no significant changes in the viable counts or in the amount of the attached biomass during the first $2 \mathrm{~h}$ in a humidified incubator. As expected, the initial attachment phase was followed by proliferation of the cells, characterized by a steady increase in the viable counts. Also, a prominent accumulation phase was observed as a time-shift in the biomass accumulation: the amount of the biomass attached to the coupons, measured by CV staining, continued to increase after $24 \mathrm{~h}$, when the number of the CFUs had already reached a maximum

Table 1

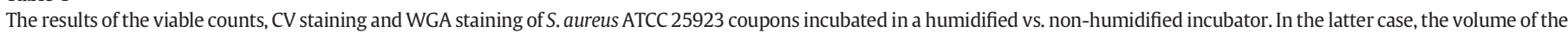

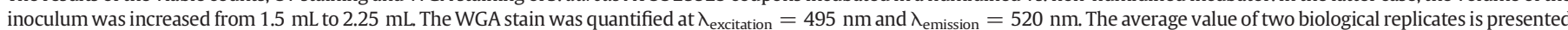

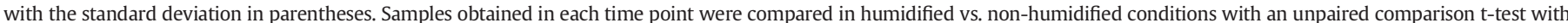
Welch's correction $(p<0.05)$ (GraphPad Prism program, San Diego, USA).

\begin{tabular}{|c|c|c|c|c|c|c|}
\hline \multirow[t]{2}{*}{ Time $(\mathrm{h})$} & \multicolumn{2}{|l|}{$\log \left(\mathrm{CFU} / \mathrm{cm}^{2}\right)$} & \multicolumn{2}{|c|}{ CV staining $\left(\mathrm{A}_{595}\right)$} & \multicolumn{2}{|c|}{ WGA staining (fluorescence) } \\
\hline & Humidified & No humidification & Humidified & No humidification & Humidified & No humidification \\
\hline 1 & $5.55( \pm 0.24)$ & $5.84( \pm 0.06)$ & $0.14( \pm 0.00)$ & $0.14( \pm 0.01)$ & $0.23( \pm 0.05)$ & $0.44( \pm 0.08)$ \\
\hline 2 & $5.77( \pm 0.03)$ & $5.94( \pm 0.02)$ & $0.14( \pm 0.01)$ & $0.14( \pm 0.00)$ & $0.46( \pm 0.25)$ & $0.68( \pm 0.25)$ \\
\hline 4 & $7.36( \pm 0.03)$ & $7.38( \pm 0.22)$ & $0.20( \pm 0.04)$ & $0.17( \pm 0.00)$ & $1.89( \pm 0.25)$ & $2.49( \pm 0.88)$ \\
\hline 24 & $8.23( \pm 0.62)$ & $7.61( \pm 0.18)$ & $0.58( \pm 0.30)$ & $0.94( \pm 0.27)$ & $9.68( \pm 1.31)$ & $9.17( \pm 2.24)$ \\
\hline 32 & $8.08( \pm 0.33)$ & $7.75( \pm 0.09)$ & $0.85( \pm 0.09)$ & $1.16( \pm 0.35)$ & N.a. & N.a. \\
\hline 48 & $8.01( \pm 0.25)$ & $7.49( \pm 0.30)$ & $1.31( \pm 0.54)$ & $1.74( \pm 0.48)$ & $12.03( \pm 4.28)$ & $11.83( \pm 1.77)$ \\
\hline
\end{tabular}

N.a. $=$ not analyzed 

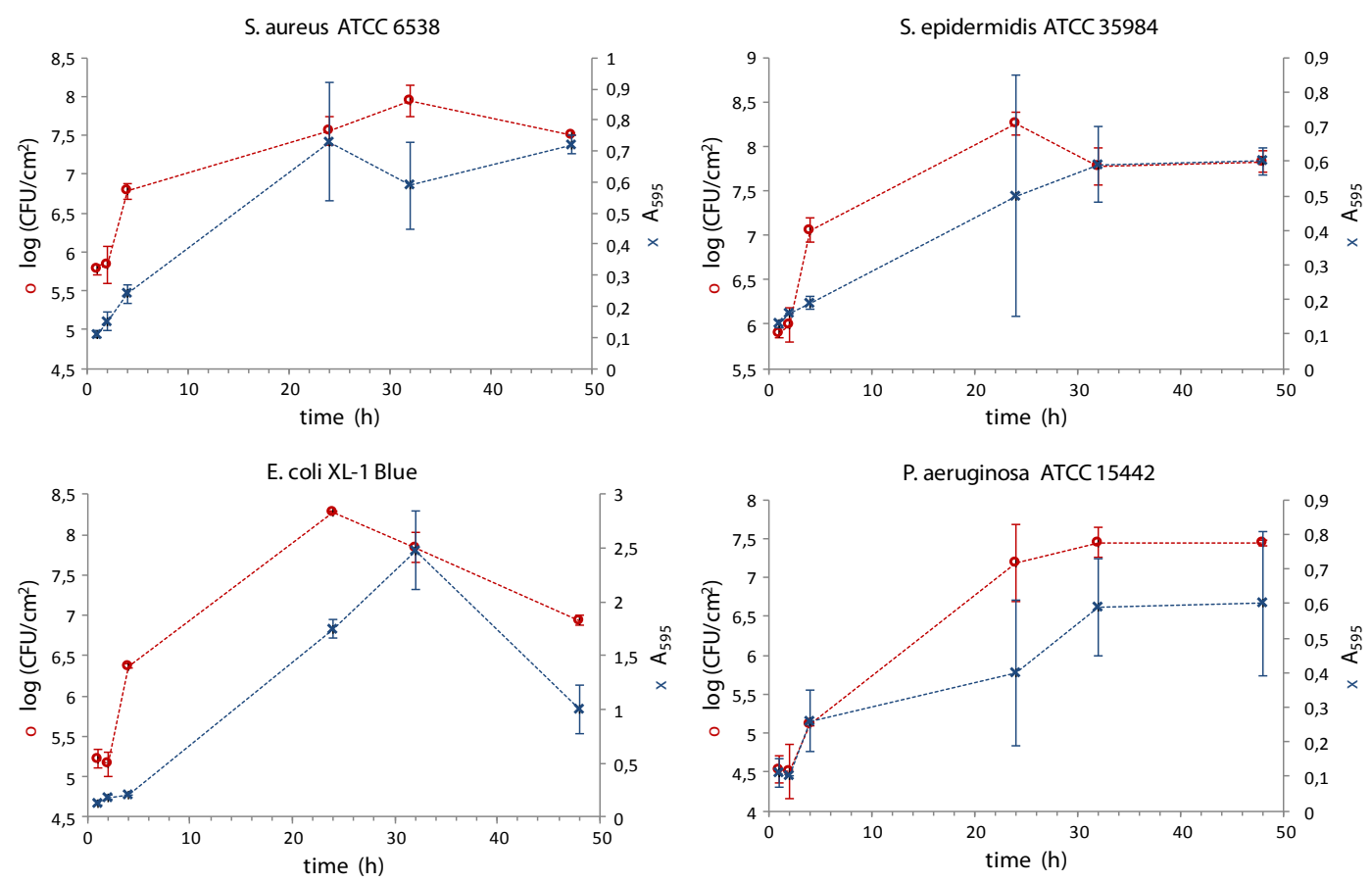

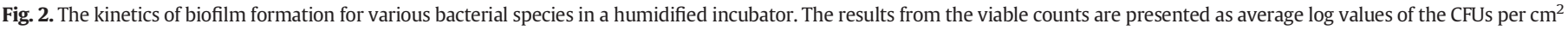

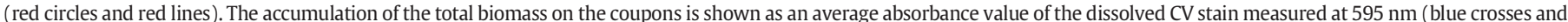

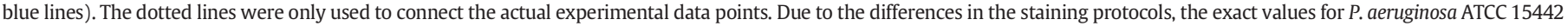

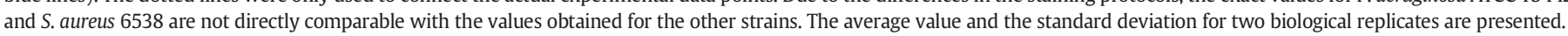

level. The accumulation of the extracellular substances was quantified by staining with the WGA conjugate that binds to the (poly-) Nacetylglucosamine residues (PNAG, Sharon, 2007). The PNAG content continued to increase after $24 \mathrm{~h}$, with a similar kinetics to the registered for the biomass, measured by CV staining. Thus, the CV and PNAG staining data support that although the cell numbers remained stable after $24 \mathrm{~h}$, the biomass that the cells were producing continued to increase. Furthermore, the possibility of using a non-humidified incubator was also evaluated. The humidification did not cause any statistically significant change in the kinetics of biofilm development, suggesting that the higher inoculation volume $(2.25 \mathrm{~mL})$ and the remoistening of the plates likely compensate for the lack of humidification (Table 1).

The results indicate that in addition to its use in the analysis of established biofilms, the static biofilm method could also be useful in the analysis of the early stages of biofilm formation, e.g. adhesion studies on various materials. The removal and dispersal of the biofilms by a simple sonication step enables easy analysis of sample coupons of varying sizes and shapes. Further flexibility is provided by the choice of the incubator: established S. aureus biofilms are formed in $48 \mathrm{~h}$ with or without humidification (Table 1).

A major advantage of the static biofilm method is the flexibility, which includes its usefulness with different bacterial species. A similar 2-h attachment period was observed in all cases, when the kinetics of biofilm formation were monitored for another $S$. aureus strain and three other bacterial species in a humidified incubator. However, some variation was seen in the length of the subsequent phase: the highest viable counts were observed at $24 \mathrm{~h}$ with $E$. coli, S. aureus ATCC 25923 and S. epidermidis, while a 32-h incubation period was necessary for the maximal counts of S. aureus ATCC 6538 and P. aeruginosa ATCC 15442 . Interestingly, with E. coli the biomass accumulation between 24 and $32 \mathrm{~h}$ was followed by a significant decline in both the attached biomass and the viable counts after $32 \mathrm{~h}$ (Fig. 2). Thus, it seems that the length of the incubation period has to be optimized for each strain.
Despite the robustness and the flexibility of the static biofilm method, there are obvious limitations related to its applicability. Since the biofilms are formed under no fluid shear, the method can only provide useful models of biofilms that are involved in, for instance, the infections of the ear and the skin. However, there are also numerous benefits related to the use of the method. It is simple and economical to use, and suitable for any laboratory equipped for basic microbiology work, which makes it a very convenient investigational tool for a wider audience. The method can be directly applied for studies on bacterial adhesion and biofilm formation on various materials. It is very flexible in terms of composition, size and shape of the test coupons, and it is currently used for material testing in our laboratory.

The authors acknowledge the financial support provided by the Academy of Finland projects WoodyFilm and ArtFilm (decision numbers 282981 and 272266), as well as by the Drug Discovery and Chemical Biology network of Biocenter Finland. D. G. was supported by a Core Fulbright Scholar Grant from the Fulbright Center in Helsinki, Finland.

\section{References}

Anderl, J.N., Franklin, M.J., Stewart, P.S., 2000. Role of antibiotic penetration limitation in Klebsiella pneumoniae biofilm resistance to ampicillin and ciprofloxacin. Antimicrob. Agents Chemother. 44, 1818-1824.

ASTM International, 2013. E2871-13. Standard Test Method for Evaluating Disinfectant Efficacy against Pseudomonas aeruginosa Biofilm Grown in CDC Biofilm Reactor using Single Tube Method. Annual Book of ASTM Standards vol. 11.05. ASTM International, West Conshohocken, PA.

Buckingham-Meyer, K., Goeres, D.M., Hamilton, M.A., 2007. Comparative evaluation of biofilm disinfectant efficacy tests. J. Microbiol. Methods 70, 236-244.

Charaf, U.K., Bakich, S.L., Falbo, D.M., 1999. A model biofilm for efficacy assessment of antimicrobials versus biofilm bacteria. Biofilms. The Good, The Bad and The Ugly. Biofilm Club, Cardiff University, UK 0-9520432-6-2, pp. 171-177.

Coenye, T., Nelis, H.J., 2010. In vitro and in vivo model systems to study microbial biofilm formation. J. Microbiol. Methods 83, 89-105.

Cos, P., Toté, K., Horemans, T., Maes, L., 2010. Biofilms: an extra hurdle for effective antimicrobial therapy. Curr. Pharm. Des. 16, 2279-2295.

Dunne Jr., W.M., 2002. Bacterial adhesion: seen any good biofilms lately? Clin. Microbiol. Rev. 15, 155-166. 
Kiedrowski, M.R., Horswill, A.R., 2011. New approaches for treating staphylococcal biofilm infections. Ann. N. Y. Acad. Sci. 1241, 104-121.

Otto, M., 2008. Staphylococcal biofilms. Curr. Top. Microbiol. Immunol. 322, 207-228.

Sandberg, M., Määttänen, A., Peltonen, J., Vuorela, P.M., Fallarero, A., 2008. Automating a

96-well microtitre plate model for Staphylococcus aureus biofilms: an approach to screening of natural antimicrobial compounds. Int. J. Antimicrob. Agents 32, 233-240.
Sharon, N., 2007. Lectins: carbohydrate-specific reagents and biological recognition molecules. J. Biol. Chem. 282, 2753-2764.

Skogman, M.E., Vuorela, P.M., Fallarero, A., 2012. Combining biofilm matrix measurements with biomass and viability assays in susceptibility assessments of antimicrobials against Staphylococcus aureus biofilms. J. Antibiot. 65, 453-459. 